\title{
An Upgraded Sine Cosine Algorithm for Tower Crane Selection and Layout Problem
}

\author{
Ali Kaveh $^{1 *}$, Yasin Vazirinia ${ }^{1}$ \\ 1 School of Civil Engineering, Iran University of Science and Technology, Narmak, Tehran, P.O. Box 16846-13114, Iran \\ ${ }^{*}$ Corresponding author, e-mail: alikaveh@iust.ac.ir
}

Received: 30 November 2019, Accepted: 30 January 2020, Published online: 27 February 2020

\begin{abstract}
Tower crane is the core construction facility in the high-rise building construction sites. Proper selection and location of construction tower cranes not only can affect the expenses but also it can have impact on the material handling process of building construction. Tower crane selection and layout problem (TCSLP) is a type of construction site layout problem, which is considered as an NP-hard problem. In consequence, researchers have extensively used metaheuristics for their solution. The Sine Cosine Algorithm (SCA) is a newly developed metaheuristic which performs well for TCSLP, however, efficient use of this algorithm requires additional considerations. For this purpose, the present paper studies an upgraded sine cosine algorithm (USCA) that employs a harmony search based operator to improve the exploration and deal with variable constraints simultaneously and uses an archive to save the best solutions. Subsequently, the upgraded sine cosine algorithm is employed to optimize the locations to find the best tower crane layout. Several benchmark functions are studied to evaluate the performance of the USCA. A comparative study indicates that the USCA performs quite well in comparison to other recently developed metaheuristic algorithms.
\end{abstract}

\section{Keywords}

Tower Crane Layout, Upgraded Sine Cosine Algorithm, construction site layout, global optimization, local search, tower crane selection

\section{Introduction}

Tower cranes are fundamental components in lifting heavy and colossal items at construction sites because of being versatile tools. They can handle objects, including steel beams, prefabricated components, mixed concrete, and heavy tools such as equipment and various machinery, to name but a few. On the other hand, recent improvements in technologies provided new opportunities to increase the use of prefabrication and modularization in large buildings [1]. According to the assembly speed of these structures in the construction process, the transportation of prefabricated elements is remarkably essential. In terms of safety and accessibility, the location of tower cranes is extremely important for being capable of handling both colossal and heavy materials on the site. In fact, the selection of tower cranes location can be of great importance in the total efficiency of a construction site because it has overlap with the Construction Site Layout Problems (CSLPs). In order to meet their final goals such as dropping various construction materials between demand and supply points, reaching and covering their job in a way that it can cover all necessary parts of the buildings in the site is a necessary prerequisite. Subsequently, for any successful tower crane locating, some considerations such as transportation distances and operating costs are supposed to be taken into account. In this vein, the tower crane layout should be carefully optimized to meet the above-mentioned goals. Therefore, the better layout of both tower cranes and the locations of material supply we have, the more productive efficiency we will have in construction sites.

Moreover, tower crane layout planning (TCLP) is considered as a combinatorial optimization problem [2]. Clearly, in the last few decades, much great research has been conducted so that researchers can figure out the best way of approaching combinatorial Construction Engineering Optimization Problems (CEOPs). As a result of which, researchers have extensively used metaheuristics in order to uncover the tower crane selection and layout problems. Metaheuristics are well-known and practical methods for solving complex optimization problems. These algorithms optimize iteratively by mimicking the 
biological evolution, artificial intelligence, nervous systems, statistical mechanics, mathematical and physical sciences, and classic heuristics so that the results approach the optimal solutions [3]. The Sine Cosine Algorithm (SCA) [4] is one of the recently developed optimization techniques inspired by the sine and cosine mathematical functions. Although there are several techniques to solve TCSLP before, they suffer problems like low convergence speed and easily fell into the local optima. On the other hand, SCA is a new type of algorithm to encounter this problem. Since the SCA has poor stability, and the experimental results also have plenty of room for optimization, therefore, this paper proposes an algorithm, called Upgraded Sine Cosine Algorithm (USCA), that has better stability and faster convergence than the original SCA. This algorithm employs the SCA pattern and updates it considering the above points. For this purpose, a memory is added to save the best agents and a harmony based side constraint handling approach is utilized. Incorporating these approaches a new variant of SCA, namely USCA, is proposed to solve the TCSLP. The experimental results intimate that USCA provides better performance than its standard version.

Section 2 presents a brief review of the related works; Section 3 explains the optimization algorithms. Some optimization problems are described in Section 4. Experimental studies are presented in Section 5, and the results are discussed, and conclusions are derived in Section 6.

\section{Literature review}

In this research, the topics of Metaheuristic Algorithms and Sine Cosine Algorithm (SCA), besides Tower Crane selection and layout problem is meticulously elaborated upon in the following paragraphs.

\subsection{Metaheuristic algorithms}

In the last few decades, there has been a considerable growing interest in metaheuristic algorithms in order to discover better solutions for problems involved in our daily lives. As a result of this, a verity of metaheuristics - with various attitudes and aspects - are developed, and at the same time, they are utilized in virtually all fields. Efficiency is one of the main goals of these optimization methods, which can eventually lead to a global solution. These algorithms are neither problem-specific nor depend on the derivatives of the objective functions. The industry and academic community are tremendously paying attention to this field of knowledge [5]. Being a global method, metaheuristic methods trying to stimulate natural phenomena (particle swarm optimization [6], genetic algorithm [7], ant lion optimizer (ALO) [8], Cyclical Parthenogenesis Algorithm (CPA) [9]), socio-cultural behaviors (socio evolution and learning optimization (SELO) [10] and Ideology Algorithm (IA) [11]), or physical phenomena (colliding bodies optimization [5], gravitational search algorithm (GSA) [12], charged system search (CSS) [13]). Metaheuristic optimization methods have two unique, distinctive aspects: exploration and exploitation. Exploitation focuses on finding the best available solutions and the best likely points; it also grants optimizers to scrutinize the search space, usually by randomization, in a highly efficient way. Exploitation involves generating diverse solutions for exploring the search space globally [5]. Mirjalali [4] introduced the sine cosine algorithm (SCA) based on mathematical formulations of sine and cosine functions, and this algorithm is applied to various fields of optimization widely. Previous studies have shown that SCA is able to yield encouraging results, in comparison with some other metaheuristic algorithms. Moreover, SCA, among other metaheuristic algorithms, has proven to be a promising method for resolving across different engineering and scientific problems.

\subsection{Tower crane selection and layout problem}

During the last few decades, researchers have been obsessed with finding the best method to address problems related to the Construction Engineering Optimization Problems (CEOPs) [14]. Since the main function of tower cranes are for transporting bulky construction materials [15], and also material transportation is a complex activity during the building construction process; thus, hoisting and lifting bulky materials needs meticulous planning [16]. As a result, during the last twenty years, TCLP is applied as a method to find out the best possible location for supply points and tower cranes within a building construction site to enable to meet minimum time objectives efficiently and effectively. Zhang et al. [17] expanded an analytic model taking into account the hook traversal time and then selecting a Monte Carlo simulation for optimizing the tower crane's location. Nevertheless, their assumption was based on a single crane, and also, the impact of supply points location on lifting requirements without taking into consideration the travel time.

Tam and Tong [18] have utilized an artificial neural network model in order to anticipate tower crane operations. In this vein, they also applied a model based on a genetic algorithm for optimizing the layout of tower crane and 
supply points [19]. The approach adopted by Tam et al. [19] afterward was utilized in quite a few papers to show the effectiveness of their models. For example, Huang et al. [16] applied a mixed-integer linear programming (MILP) for optimizing the tower crane and supply points location, Kaveh and Vazirinia [20] have made a comparison between the performance of physical inspired algorithms on this model and have discussed the results.

Lien and Cheng [2] utilized a model similar to Huang et al. [16] but with a different solution approach employing particle bee algorithm. Also, they expanded Huang's single tower crane model to a model with a predetermined number of tower cranes. Wang et al. [21] integrated the firefly algorithm with building information model (BIM) for solving the tower crane selection and layout problem by the objective of minimum cost-weighted hook traversal time. In addition, Marzouk and Abubakr [22] incorporated the AHP to select the best tower cranes and the Genetic Algorithm for minimizing the total operation cost of the tower crane. Karan and Irizarry [23] combined the application of the GIS and BIM for arranging tower cranes with the objective of minimal conflict.

\section{Formulation of optimization algorithms}

\subsection{Metaheuristic algorithm}

The Sine Cosine Algorithm (SCA) is proven to have a lot of capabilities which are as follow: to explore various areas in the search spaces, to exploit likely areas of the search space while optimizing efficiently, for converging to the global optimum, and also escape from the local optima, to name but a few [4]. The SCA initiates with a set of random solutions and moves toward or outwards the best solution using sine and cosine functions. Whenever the functions of sine and cosine have a value smaller than -1 or more than 1, various areas in the search space will be considered. Additionally, if the process returns the value between -1 and 1 from sine and cosine, promising areas of the search space will be exploited. As for SCA, the number of parameters - which are required to be optimized - bring about defining the dimension of the search space. The user determines the number of search agents. The current solutions have randomly initialized positions $\left(X_{i}\right)$ that will be adapted to the former positions by Eq. (1) to guarantee that the solutions constantly will have positions updated according to the optimum solution have been achieved.

$X_{i}^{i+1}=\left\{\begin{array}{l}X_{i}^{t}+r_{1} \sin \left(r_{2}\right)\left|r_{3} P_{i}^{t}-X_{i}^{t}\right|, r_{4}<0.5 \\ X_{i}^{t}+r_{1} \cos \left(r_{2}\right)\left|r_{3} P_{i}^{t}-X_{i}^{t}\right|, r_{4} \leq 0.5\end{array}\right.$
In order to make the process of convergence and divergence in the search agents smooth, four variables - random and adaptive variables - are combined. As a result of this, the balance of exploration and exploitation holds in getting the best result of regions of the research space. Finally, a globally-acceptable result can be obtained. By doing so, the range of sine and cosine will be adjustable according to the definition of the parameter $r_{1}$ in the Eq. (2). Hence, the parameter $r_{1}$ indicates the region of next position (or movement orientation), so the result likely would be either outside of the space, which is between destination and solution or inside it.

Since sine and cosine occur in a cyclic form, it enables solution to be positioned again along another solution; it explains the space exploitation between two solutions. By altering the domain of sine and cosine functions, the solutions should be able to explore the outside search space between their corresponding destinations. If we have a randomly-selected number for $r_{2}$ in range [0 2 $2 \pi$ ] in Eq. (1), the random location is obtainable for both inside and outside. Thus, the random parameter $r_{2}$ explains the distance of movement outwards or towards the destination. By doing so, this process makes certain that the search space of exploitation and exploration can be separate. The random parameter $r_{3}$ assigns a random weight to the destination for emphasizing $\left(r_{3}>1\right)$ or deemphasizing $\left(r_{3}>1\right)$ the influence of destination on defining the distance. Ultimately, the parameter $r_{4}$ changes equally among the components of sine and cosine in Eq. (1).

$r_{1}=a-t \frac{a}{T}$

Furthermore, Algorithm 1 shows the pseudo-code of the SCA algorithm. The process of optimization in the SCA begins through a set of randomly generated solutions. Subsequently, the best-achieved solutions up to now are saved by the algorithm; the algorithm determines it just as the best destination point and provides up-to-date solutions accordingly. At the same time, the domain functions of sine and cosine are brought up to date, so that the exploitation

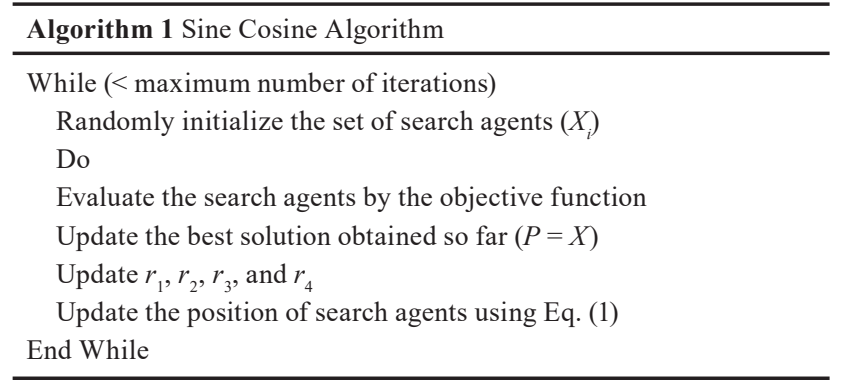


related to search space can be emphasized whenever the iteration counter goes up. The SCA algorithm automatically brings the optimization process to an end as the highest iteration number is lower than the iteration counter, vice versa. The details have been elaborated in [4].

\subsection{Upgraded Sine Cosine Algorithm}

The prominent aim of this section is introducing an upgraded version for the SCA, Upgraded Sine Cosine Algorithm (USCA), which improves the SCA getting faster with more reliable solutions. By adding the best agents memory (AM), the convergence speed of USCA can be increased with respect to the standard SCA. Moreover, changing violated components of search agents in the case of boundary violation using a side constraint handling approach based on harmony search helps the USCA in escaping from local optima [24]. The flowchart of the USCA is presented in Fig. 1, and the processes associated with the enhancement of SCA are elaborated in the following:

\section{Step 1: Initialization}

First, in the USCA, parameters will set, and then the initial locations of the agents (solutions) are randomly determined in the search space.

Step 2: Solution evaluation

According to each agent, the process starts calculating the objective function value.

\section{Step 3: Saving}

Enhancing the performance of algorithm without escalating the computational cost can be achieved through considering a memory for saving some of the historical-best search agents and regarding their objective function values [13]. In this vein, the best agents memory (AM) should be introduced, for saving some of the best solutions up to now. Then, AM members will be used as destination agents randomly.

Step 4: Updating the positions of the agents

According to the sine cosine concept, the positions are updated by Eq. (1).

Step 5: Side constraints handling

Though by moving the agent in the search space, a better solution can be obtained, still there is a possibility to violate the side constraints. Common side constraint handling approaches may lessen the exploration capability of the algorithm. Moreover, during the optimization process, it is important to balance exploration and exploitation. Regarding these issues, a harmony search-based side constraint-handling approach is utilized to regenerate the violated components $[13,24]$. As for this method, in order to identify whether the violated component should be altered with the equivalent component of a random AM member with AMCR (Agent Memory Considering Rate) probability (in range [0 1]), or it has to be determined randomly within the search space by the probability of (1-AMCR).

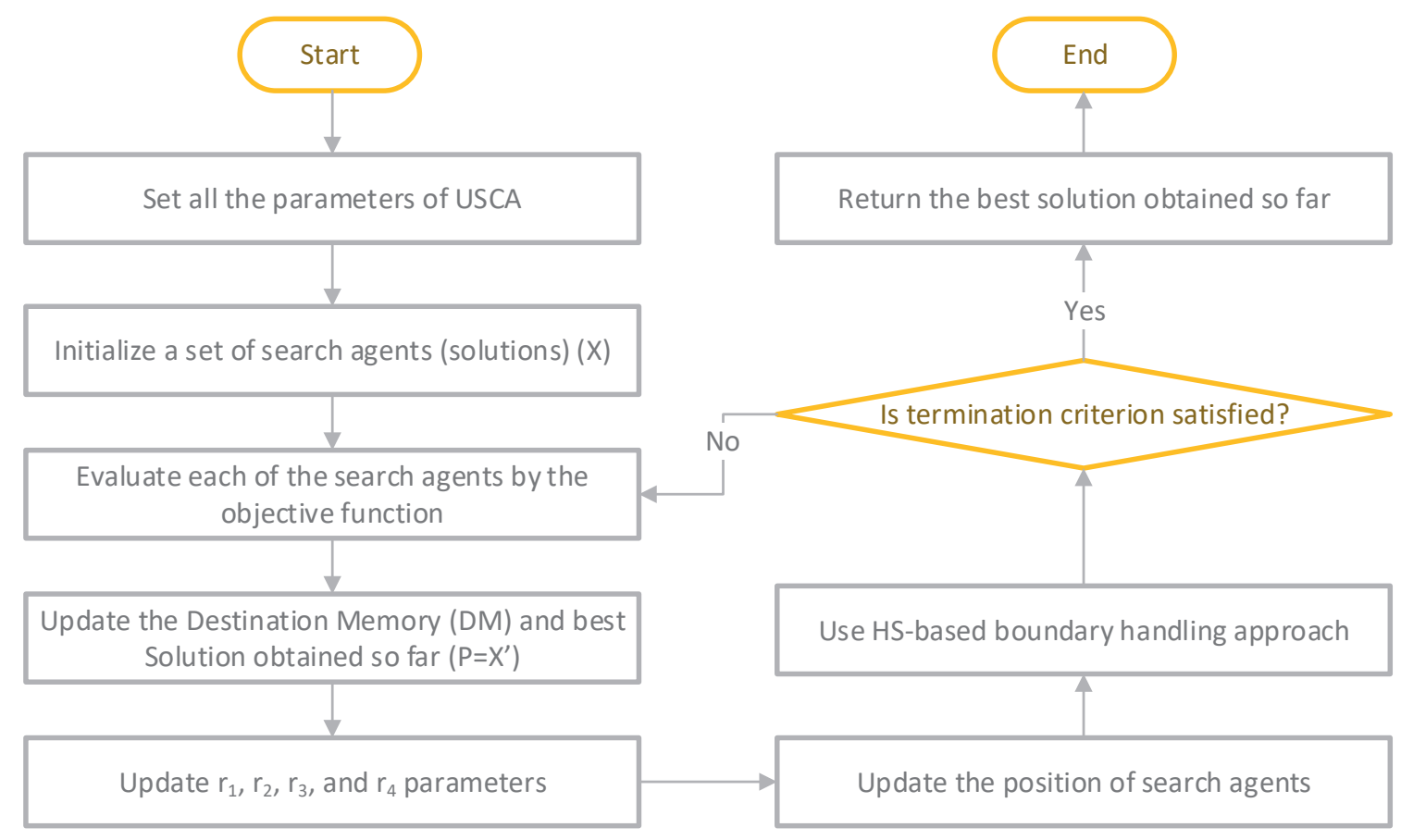

Fig. 1 Flowchart of the proposed USCA algorithm 
In addition, still, when the component of an AM member is chosen, we have a possibility such as Pitch Adjusting Rate (PAR) specifying whether this value needs to be altered with a neighboring value or not. If a value is chosen from the AM, the pitch adjusting process will be performed. The value of (1-PAR) sets inaction rate, and PAR adjusts the rate of selecting a value from the neighboring of the best AM. Algorithm 2 shows the process of handling side constraints. The readers may refer to [13, 24] for additional details.

\section{Step 6: Terminating condition check}

The process of optimization ends following a fixed number of iterations. If this criterion does not meet its goal, steps 2 to 6 will be repeated for another round of iteration.

As far as this study is concerned, any condition can be considered for termination and here the optimization process terminates after fixed number of iterations.

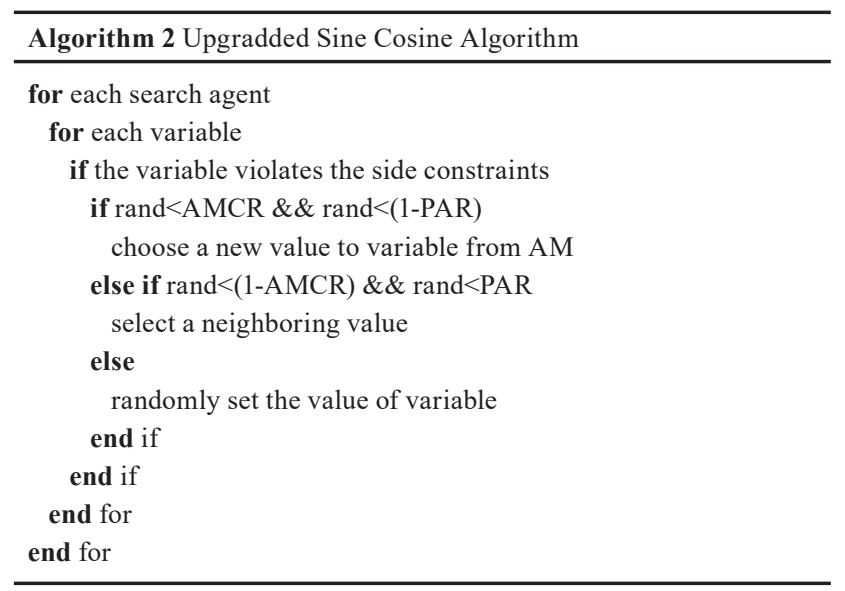

\section{Optimization problems}

In metaheuristic optimization, many test cases are usually applied to illustrate the performance of algorithms because of the stochastic nature of these algorithms. There is an adequate collection of test functions; therefore, a group of models should be applied to ascertain that the best findings do not happen by chance. Nevertheless, still, there is a lack of a vivid definition of suitability for a set of benchmark case studies. Thus, this research tried to evaluate the USCA algorithm on mathematical test functions with various characteristics. The set of test problems utilized here encompasses three groups: uni-modal and multi-modal test functions, and TCSLP. Then, three real-sized TCSLP case studies are solved by the ASCA algorithm as well.

\subsection{Mathematical test functions}

Tables 1 and 2 present the formulation of the mathematical test functions. There is just one global optimum without any local optima in the first family of test functions. This makes them very suitable to test the exploitation and convergence speed of algorithms. The other group of test functions, has although multiple local optima along with a globally optimum solution. These characteristics are advantageous for getting the explorative ability of an algorithm and testing local optima avoidance. The ASCA algorithm is superior to its standard version and some well-known algorithms like Whale Optimization Algorithm (WOA), Vibrating Particles System (VPS), Slap Swarm Optimization (SSA), Colliding Bodies Optimization (CBO), and PSO for verification of the results.

Table 1 Uni-modal test functions

\begin{tabular}{|c|c|c|c|}
\hline Function & Shift position & Dim & $f_{\min }$ \\
\hline$F_{1}(x)=\sum_{n=1}^{N} x_{n}^{2}$ & {$[-100,100]$} & 30 & 0 \\
\hline$F_{2}(x)=\sum_{n=1}^{N}\left|x_{n}\right|+\prod_{n=1}^{N}\left|x_{n}\right|$ & {$[-10,10]$} & 30 & 0 \\
\hline$F_{3}(x)=\sum_{n=1}^{N}\left(\sum_{n-1}^{N} x_{n}\right)^{2}$ & {$[-100,100]$} & 30 & 0 \\
\hline$F_{4}(x)=\max _{n}\left\{\left|x_{n}\right|, 1 \leq n \leq N\right\}$ & {$[-100,100]$} & 30 & 0 \\
\hline$F_{5}(x)=\sum_{n=1}^{N-1}\left[100\left(x_{n+1}-x_{n}^{2}\right)^{2}+\left(x_{n}-1\right)^{2}\right.$ & {$[-30,30]$} & 30 & 0 \\
\hline$F_{6}(x)=\sum_{n=1}^{N}\left(\left[x_{n}+0.5\right]\right)^{2}$ & {$[-100,100]$} & 30 & 0 \\
\hline$F_{7}(x)=\sum_{n=1}^{N} n x_{n}^{4}+\operatorname{random}\left[\begin{array}{ll}0 & 1\end{array}\right]$ & {$[-1.28,1.28]$} & 30 & 0 \\
\hline
\end{tabular}


Table 2 Multi-modal test functions

\begin{tabular}{|c|c|c|c|}
\hline Function & Shift position & Dim & $f_{\min }$ \\
\hline$F_{8}(x)=\sum_{n=1}^{N}-x_{n} \sin \left(\sqrt{\left|x_{n}\right|}\right)$ & {$[-500,500]$} & 30 & $-418.9829 \times 5$ \\
\hline$F_{9}(x)=\sum_{n=1}^{N}\left[x_{n}^{2}-10 \cos \left(2 \pi x_{n}\right)+10\right]$ & {$[-5.12,5.12]$} & 30 & 0 \\
\hline$F_{10}(x)=-20 \exp \left(-0.2 \sqrt{\frac{1}{n} \sum_{n=1}^{N} x_{n}^{2}}\right)-\exp \left(\frac{1}{N} \sum_{n=1}^{N} \cos \left(2 \pi x_{n}\right)\right)+20+e$ & {$[-32,32]$} & 30 & 0 \\
\hline$F_{11}(x)=\frac{1}{4000} \sum_{n=1}^{N} x_{n}^{2}-\prod_{n=1}^{N} \cos \left(\frac{x_{n}}{\sqrt{n}}\right)+1$ & {$[-600,600]$} & 30 & 0 \\
\hline \multicolumn{4}{|c|}{$F_{12}(x)=\frac{\pi}{N}\left\{10 \sin \left(\pi y_{1}\right)+\sum_{n=1}^{N-1}\left(y_{n}-1\right)^{2}\left[1+10 \sin ^{2}\left(\pi y_{n+1}\right)\right]+\left(y_{N}-1\right)^{2}\right\}+\sum_{n=1}^{N} u\left(x_{n}, 10,100,4\right)$} \\
\hline$y_{n}=1+\frac{x_{n}+1}{4} u\left(x_{n}, a, k, m\right)=\left\{\begin{array}{cc}k\left(x_{n}-a\right)^{m} & x_{n}>a \\
0 & -a<x_{n}<a \\
k\left(-x_{n}-a\right)^{m} & x_{n}<-a\end{array}\right.$ & {$[-50,50]$} & 30 & 0 \\
\hline \multicolumn{4}{|c|}{$F_{13}(x)=0.1 \times\left\{\sin ^{2}\left(3 \pi x_{1}\right)+\sum^{N}\left(x_{n}-1\right)^{2}\left[1+\sin ^{2}\left(3 \pi x_{n}+1\right)\right]+\left(x_{N}-1\right)^{2}\left[1+\sin ^{2}\left(2 \pi x_{N}\right)\right]\right\}$} \\
\hline$+\sum_{n=1}^{N} u\left(x_{n}, 5,100,4\right)$ & & & \\
\hline
\end{tabular}

\subsection{Tower crane selection and layout problem (TCSLP)}

The workspace of construction sites located in the urban context is usually very limited, and the spaces for material storage are comparably small. In this section, the mathematical formulation of a constrained tower crane selection and layout problem (TCSLP) with discrete and continuous variables are investigated to demonstrate the efficiency of the USCA algorithm, incorporating the sine cosine algorithm. In order to figure out the best approach in selecting the proper tower crane and finding the best layout, a number of instances have been studied. In this model, a single tower crane transfers materials from the optimized location of supply yard to the demand points. The mathematical formulation and constraints are presented in Eqs. (3)-(19):

\subsubsection{Hook movement time}

Having a calculated total material transportation time by a tower crane, the movement time of the hook is an important parameter. Therefore, in order to have an accurate time parameter, the hook traversal time is divided up into vertical and horizontal paths to show all operation cost. Figs. 2 and 3 illustrate the comparable movement path with various directions. Travel span, which is a distance between demand points and supply, is measured through Eqs. (3)-(5) referring to Figs. 2 and 3.

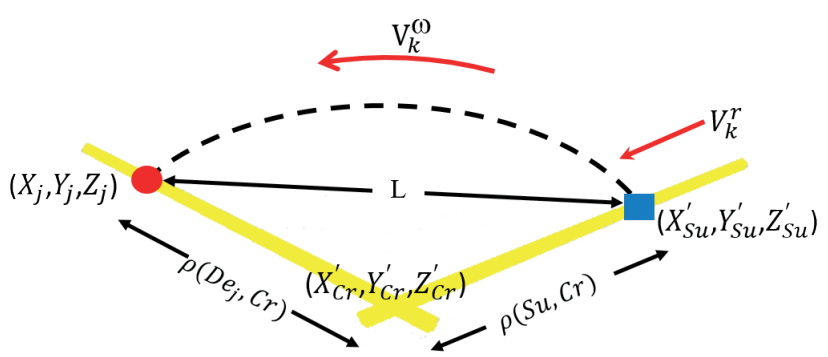

Fig. 2 Radial and tangential movements of the crane hook
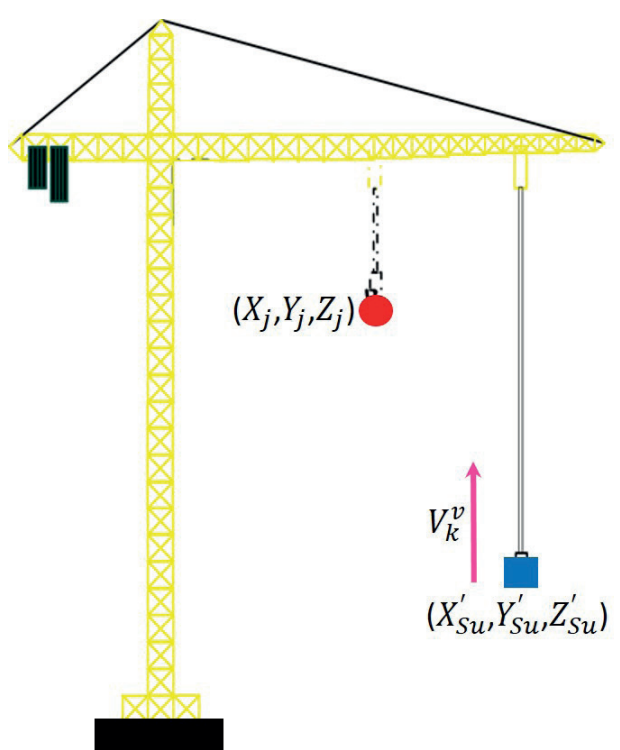

Fig. 3 Vertical movement of the crane hook 


$$
\begin{aligned}
& \rho\left(D e_{j}, C r\right)=\sqrt{\left(X_{j}-X_{C r}^{\prime}\right)^{2}+\left(Y_{j}-Y_{C r}^{\prime}\right)^{2}} \\
& \rho(S u, C r)=\sqrt{\left(X_{S u}^{\prime}-X_{C r}^{\prime}\right)^{2}+\left(Y_{S u}^{\prime}-Y_{C r}^{\prime}\right)^{2}} \\
& L=\sqrt{\left(X_{S u}^{\prime}-X_{j}\right)^{2}+\left(Y_{S u}^{\prime}-Y_{j}\right)^{2}}
\end{aligned}
$$

The continuous type parameter " $\alpha$ " - which has to do with the tower crane operator's capability in controlling it - specifies the degree of hook movement coordination in tangential and radial orientations. As a result of this, the time of both vertical and horizontal movements of hook is computed in Eqs. (8) and (9), respectively.

$$
\begin{aligned}
& T_{j, k}^{r}=\frac{\left|\rho\left(D e_{j}, C r\right)-\rho(\mathrm{Su}, \mathrm{Cr})\right|}{V_{k}^{r}} \\
& T_{j, k}=\frac{1}{V_{k}} \cos ^{-1}\left[\frac{L^{2}-\rho\left(D e_{j}, C r\right)^{2}-\rho(S u, C r)^{2}}{-2 * \rho\left(D e_{j}, C r\right) * \rho(S u, C r)}\right], \\
& 0<\cos ^{-1}(\theta)<\pi \\
& T_{j, k}^{h}=\max \left\{T_{j, k}^{r}+T_{j, k}\right\}+\alpha^{*} \min \left\{T_{j, k}^{r}+T_{j, k}\right\} \\
& T_{j, k}^{v}=\frac{\left|Z_{j}^{D e}-Z^{S u}\right|}{V_{k}^{v}}
\end{aligned}
$$

For the tower crane, all the travel time between supply area and demand point $j$ through the tower crane type $k$ can be estimated by applying Eq. (10), $T_{j, k}$, this result is achievable by defining the continuous parameter called $\beta$ which is necessary for defining the coordination degree of both horizontal and vertical planes according to the hook's movement.

$$
T_{j, k}=\lambda \cdot\left[\max \left\{T_{j, k}^{h}+T_{j, k}^{v}\right\}+\beta \cdot \min \left\{T_{j, k}^{h}+T_{j, k}^{v}\right\}\right]
$$

It is worth bearing in mind that the hook functioning properly and changing the location of tower crane are greatly affected by the state of circumstances such as the level of operator's proficiency and the level of visibility of surrounding due to the climate; so, these factors can probably decrease the overall efficiency as well. In other words, the more operation takes time, the more likely the tower crane should be moved to another place [16].

If something does not allow the operator to see properly, the total travel time needs to be increased accordingly. In this regard, another numerical parameter $\lambda$ should be taken into account when it comes to the total time of hook travel time and tower crane, see Eq. (10). Various $\lambda$ needs to be utilized for different locations of crane $k$ for determining location-specific effects in a construction site. Having high-tech vision tools in tower cranes help operators to see better and at the same time help the operation to carry out faster which means a smaller $\lambda$ is applicable [25].

\subsubsection{Objective function}

The TCSLP is formulated as a mixed-integer nonlinear programming (MINLP) facility layout design problem (FLDP). The objective function (Eq. (11)) is represented as the total cost of material transportation, which includes the fixed cost of tower crane and operational cost; these costs are highly contingent upon the actual amount of materials transporting between the location of supply area and demand points. This model not only optimizes the layout of the tower crane and supply area but also considers the selection of a proper type of tower crane.

$$
\min T C=\sum_{j=1}^{J} \sum_{k=1}^{K} T_{j k} \delta_{j k} O C_{k}+\sum_{k=1}^{K} \aleph_{k} F C_{k}
$$

\subsubsection{Demand satisfaction constraints}

To make sure that every demand $j$ is served by supply point using tower crane type $k$, the constraints Eqs. (12) and (13) are employed.

$$
\begin{aligned}
& \sum_{k=1}^{K} \delta_{j k} \geq 1, \forall j \in\{1, J\} \\
& \sum_{j=1}^{J} \delta_{j k} \leq M \aleph_{k}, \forall k \in\{1, K\}
\end{aligned}
$$

\subsubsection{Assignment constraint}

Moreover, Eq. (14) guaranties the assignment of maximum one tower crane for each tower crane location.

$$
\sum_{k=1}^{K} \aleph_{k}=1
$$

\subsubsection{Capacity constraints}

In the process of proper tower crane selection and optimal layout of the tower crane and supply yard, the crane has to meet the capacity related constraints Eqs. (15) and (16). Whereby during the travel of materials from supply yard to the demand points the load moment across the jib must be less than the maximum load moment capacity (Eq. (15)), which can be approximated as the product of load (weight of demand) and distance from mast $\rho$. Also, the maximum value of demands' weight has to meet tower crane's overall capacity, which is guaranteed by Eq. (15). 
$\max \left\{\left(\delta_{j k}\right.\right.$ Weight $\left._{j} \rho(S u, C r)\right),\left(\delta_{j k}\right.$ Weight $\left.\left._{j} \rho\left(D e_{j}, C r\right)\right)\right\}$

$\leq M L M_{k} \aleph_{k}, \forall\left\{\begin{array}{l}j \in\{1, J\} \\ k \in\{1, K\}\end{array}\right.$

$\max _{j \in\{1, J\}\}}\left(\right.$ Weight $\left._{j}\right) \geq \operatorname{Cap}_{k}, \forall\{k \in\{1, K\}$

\subsubsection{Covering constraints}

All of the supply and demand points should be covered by the radius and height-under-hook of the tower crane to ensure the physical reachability of these points by the crane (Eqs. (17) and (18)).

$$
\begin{aligned}
& \max \left\{\rho(\mathrm{Su}, \mathrm{Cr}), \rho\left(D e_{j}, C r\right)\right\} \leq R a_{k}, \forall\left\{\begin{array}{l}
j \in\{1, J\} \\
k \in\{1, K\}
\end{array}\right. \\
& \max _{j \in\{1, J\}}\left(Z_{j}\right) \geq \mathrm{HUH}_{k}, \forall\{k \in\{1, K\}
\end{aligned}
$$

\subsubsection{Area size constraint}

The dimensions of all facilities (here supply yard) have to meet the given area and size requirements. This circumstance is controlled by defining the Eqs. (19) and (20).

$$
\begin{aligned}
& \frac{1}{\mu_{S u}} \leq \frac{\left(\frac{A_{S u}}{L_{S u}^{x}}\right)}{L_{S u}^{x}} \leq \mu_{S u} \\
& L_{S u}^{y}=\frac{A_{S u}}{L_{S u}^{x}}
\end{aligned}
$$

\subsubsection{Side constraints}

Various side constraints that occur in facility layout design can be included simply into TCSLP. In case that two departments (e.g., tower crane, supply yard or building blocks) should be placed distanced from each other. It may be specified that two departments should be distanced with some minimum predefined distance $\varnothing>0$; this condition is modeled by combining $\varnothing$ to the left hand side of Eqs. (21)-(23). This also can be generalized along directions $\mathrm{X}$ and $\mathrm{Y}$.

$$
\begin{aligned}
& \max \left\{\left|X_{C r}^{\prime}-X_{S u}^{\prime}\right|-\frac{L_{C r, k}^{x}+L_{S u}^{x}}{2},\left|Y_{C r}^{\prime}-Y_{S u}^{\prime}\right|-\frac{L_{C r, k}^{y}+L_{S u}^{y}}{2}\right\} \\
& \geq \max \left\{\varnothing_{C r, k}^{\min }, \varnothing_{S u}^{\min }\right\}, \forall k \in\{1, K\} \\
& \max \left\{\left|X_{C r}^{\prime}-X_{o}\right|-\frac{L_{C r, k}^{x}+L_{o}^{x}}{2},\left|Y_{C r}^{\prime}-Y_{o}\right|-\frac{L_{C r, k}^{y}+L_{o}^{y}}{2}\right\} \\
& \geq \max \left\{\varnothing_{C r, k}^{\min }, \varnothing_{o}^{\min }\right\}, \forall\left\{\begin{array}{l}
o \in\{1, O\} \\
k \in\{1, K\}
\end{array}\right.
\end{aligned}
$$

$$
\begin{aligned}
& \varnothing_{C r, k}^{\max } \geq \min _{o \in\{1, O\}}\left\{\left|X_{C r}^{\prime}-X_{o}\right|,\left|Y_{C r}^{\prime}-Y_{o}\right|\right\}, \forall k \in\{1, K\} \\
& \max \left\{\left(\left|X_{S u}^{\prime}-X_{o}\right|-\frac{L_{S u}^{x}-L_{o}^{x}}{2}\right),\left(\left|Y_{S u}^{\prime}-Y_{o}\right|-\frac{L_{S u}^{y}-L_{o}^{y}}{2}\right)\right\} \\
& \geq \max \left\{\varnothing_{S u}^{\min }, \varnothing_{o}^{\min }\right\}, \forall o \in\{1, O\}
\end{aligned}
$$

Non-rectangular departments or obstacles (building blocks) can be modeled employing well-sized fixed flawless artificial rectangular facilities (dividing buildings into rectangular departments). For modeling of the fixed departments (building blocks), only their actual width, length, and centroid should be determined, i.e., if $o$ is fixed, $\left(L_{o}^{x} ; L_{o}^{y}\right)$ and $\left(X_{o} ; Y_{o}\right)$ are known parameters. Of course, departments with fixed orientation or shape can be modelled as well.

$$
\begin{aligned}
& 0 \leq X_{C r}^{\prime}+\frac{L_{C r, k}^{x}}{2}+\varnothing_{o}^{\min } \leq L^{x}, \forall k \in\{1, K\} \\
& 0 \leq Y_{C r}^{\prime}+\frac{L_{C r, k}^{y}}{2}+\varnothing_{o}^{\min } \leq L^{y}, \forall k \in\{1, K\} \\
& 0 \leq X_{S u}^{\prime}+\frac{L_{S u}^{x}}{2}+\varnothing_{S u}^{\min } \leq L^{x} \\
& 0 \leq Y_{S u}^{\prime}+\frac{A_{S u}}{2 L_{S u}^{x}}+\varnothing_{S u}^{\min } \leq L^{y}
\end{aligned}
$$

\section{Exploratory study (results and discussion)}

For the sake of completeness of the investigations, the results of USCA is compared with several algorithms: the standard SCA algorithm, some well-known algorithms such as the PSO [6] Vibrating Particles System (VPS) algorithm [24], Colliding Bodies Optimization (CBO) [5], Whale Optimization Algorithm [26], Salp Swarm Algorithm (SSA) [27]. After several initial pilot experiments in MATLAB R2017a for determining the suitable parameter settings, the algorithms are employed to find the optimal solution.

Regarding the central limit theorem, it is a prerequisite for the sample size to be at least 30 to achieve statistically significant data. By increasing the size of a sample, its distribution converges to normal distribution [28].

Three instances of tower crane selection and layout problem have been studied in this research.

For solving the mathematical test functions, the number of search agents is set to 30 for determining the global optimum after 500 iterations. Also, to solve the TCSLP case studies 1 to 3 , the number of search agents is set to 50, after 500 iterations. 


\subsection{Results of the algorithms on mathematical test functions}

5.1.1 Results of the algorithms on uni-modal test functions Since functions F1 to F7 have just one global optimum, they are uni-modal. These functions make it possible to assess the exploitation ability of the analyzed metaheuristic algorithms. According to Table 3, USCA outperforms the rest of metaheuristic algorithms in most of the analyzed cases. Especially, it is either the most effective optimizer for F1, F2, F4, and F7 functions or at least the best second optimizer among the majority of test problems. Therefore, the current algorithm can come up with excellent exploitation.

\subsubsection{Results of the algorithms on multi-modal test functions}

In contrast to unimodal functions, multimodal functions contain many local optima, in which their number escalates rapidly with the number of variables, in other words, problem size. Subsequently, as far as the purpose is assessing the exploration capability of an optimization algorithm, this type of test problem can be handy. The findings presented in Table 3 for functions F8-F13 indicate that USCA not only outperforms SCA but also it has better exploration performance in comparison to majority of the algorithms (F9, F10, F12, and F13). This is because of integrated local search mechanisms into the SCA algorithm, which guides this algorithm with this aim for getting the global optimum.

\subsection{Results of the algorithms on Tower crane selection and layout problems}

In this section, the performance of the USCA and SCA are compared with newly developed metaheuristic algorithms (WOA and SSA) and some known metaheuristic algorithm from the literature with regard to their efficiency in resolving TCSLP. In order to explore the effectiveness of the suggested USCA algorithm on the

Table 3 Results of algorithms for the uni-modal and multi-modal benchmark functions

\begin{tabular}{|c|c|c|c|c|c|c|c|c|}
\hline & & PSO & VPS & $\mathrm{CBO}$ & WOA & SSA & SCA & USCA \\
\hline \multirow{2}{*}{$\mathrm{F} 1$} & avg & $9.48 \mathrm{E}-7$ & $5.19 \mathrm{E}-15$ & $2.44 \mathrm{E}-26$ & $3.60 \mathrm{E}-72$ & $1.54 \mathrm{E}-7$ & 0.01592 & $1.76 \mathrm{E}-74$ \\
\hline & Std & $1.2 \mathrm{E}-6$ & $2.82 \mathrm{E}-14$ & $1.32 \mathrm{E}-25$ & $1.92 \mathrm{E}-71$ & $1.81 \mathrm{E}-7$ & 0.042846 & $9.66 \mathrm{E}-74$ \\
\hline \multirow{2}{*}{$\mathrm{F} 2$} & Avg & 0.010042 & 0.067836 & $2.48 \mathrm{E}-18$ & $1.93 \mathrm{E}-51$ & $1.77 \mathrm{E}-7$ & $2.42 \mathrm{E}-5$ & $8.99 \mathrm{E}-53$ \\
\hline & Std & 0.030413 & 0.158446 & 7.79E-18 & $6.99 \mathrm{E}-51$ & 1.2525 & $5.92 \mathrm{E}-5$ & $2.94 \mathrm{E}-52$ \\
\hline \multirow{2}{*}{ F3 } & Avg & 146.8553 & 973.9987 & $1.94 \mathrm{E}-10$ & 430.016 & 165.271 & 459.4629 & 17.16201 \\
\hline & Std & 88.2275 & 340.8949 & $6.21 \mathrm{E}-10$ & 150.3572 & 79.18721 & 445.2604 & 25.2121 \\
\hline \multirow{2}{*}{$\mathrm{F} 4$} & Avg & 2.4524 & 7.910933 & 2.9034 & 40.7526 & 11.4869 & 17.6149 & $1.17 \mathrm{E}-8$ \\
\hline & Std & 0.87091 & 1.707227 & 6.2131 & 30.8293 & 3.4217 & 7.7678 & $3.47 \mathrm{E}-8$ \\
\hline \multirow{2}{*}{ F5 } & Avg & 59.2953 & 50.39215 & 11.4806 & 28.033 & 7.2484 & 629.5354 & 352.0901 \\
\hline & Std & 34.1289 & 10.64304 & 25.2131 & 0.43029 & 0.44445 & 2133.313 & 435.8026 \\
\hline \multirow{2}{*}{ F6 } & Avg & $1.7 \mathrm{E}-6$ & $8.33 \mathrm{E}-11$ & 0.33647 & 0.46688 & $2.6 \mathrm{E}-7$ & 4.5506 & 0.004756 \\
\hline & Std & $3.3 \mathrm{E}-6$ & $4.56 \mathrm{E}-10$ & 0.12368 & 0.26757 & $4 \mathrm{E}-7$ & 0.42805 & 0.016016 \\
\hline \multirow{2}{*}{ F7 } & Avg & 0.025389 & 0.011242 & 0.00157 & 0.002781 & 0.15816 & 0.54693 & 0.001307 \\
\hline & Std & 0.010805 & 0.027211 & 0.001796 & 0.003461 & 0.069296 & 0.058784 & 0.001328 \\
\hline \multirow{2}{*}{ F8 } & Avg & -6467.978 & -2269.17 & -8239.69 & -3314.42 & -10124.0 & -3924.64 & -7569.041 \\
\hline & Std & 716.6799 & 883.2805 & 473.2457 & 631.8645 & 1799.41 & 251.6031 & 821.5397 \\
\hline \multirow{2}{*}{ F9 } & Avg & 44.1098 & 31.49915 & 0.76084 & $4.73 \mathrm{E}-16$ & 53.6945 & 13.2587 & 0 \\
\hline & Std & 12.6133 & 0.062087 & 4.1673 & $2.59 \mathrm{E}-15$ & 19.6514 & 21.2695 & 0 \\
\hline \multirow{2}{*}{ F10 } & Avg & 1.1975 & 0.068006 & $1.97 \mathrm{E}-14$ & $5.15 \mathrm{E}-15$ & 2.7508 & 14.5692 & $3.61 \mathrm{E}-15$ \\
\hline & Std & 0.91833 & 0.038193 & $3.56 \mathrm{E}-14$ & $2.53 \mathrm{E}-15$ & 1.0111 & 8.4603 & $2.41 \mathrm{E}-15$ \\
\hline \multirow{2}{*}{ F11 } & Avg & 0.020682 & 30.95678 & 0.016347 & 0 & 0.019367 & 0.22649 & 0.06301 \\
\hline & Std & 0.025434 & 17.28944 & 0.046879 & 0 & 0.014123 & 0.23017 & 0.174 \\
\hline \multirow{2}{*}{ F12 } & Avg & 0.20882 & 1.302539 & 0.066466 & 0.024438 & 7.8765 & 21.887 & 0.006366 \\
\hline & Std & 0.353 & 0.559189 & 0.026203 & 0.016628 & 3.9347 & 103.5234 & 0.009402 \\
\hline \multirow{2}{*}{ F13 } & Avg & 0.052337 & 8.229969 & 0.27566 & 0.56922 & 17.1453 & 43.8061 & 0.027852 \\
\hline & Std & 0.16668 & 3.28645 & 0.095411 & 0.266 & 16.0224 & 196.1412 & 0.039896 \\
\hline
\end{tabular}

The best statistical results are shown in bold. 
TCSLP three real-sized structures presented by Kaveh and Ilchi Ghazaan [29] are used. In all of these examples, all frame members are line elements, and the height of all stories are equal to $3.5 \mathrm{~m}$. Also, the information of 72 tower crane alternatives are presented in Appendix A1. The design variables are consists of an integer variable for tower crane selection and continuous type variables to determine the location of tower crane and supply point and dimensions of supply yard. In all these cases the required area of supply yard $A_{S u}$ is equal to $40 \mathrm{~m}^{2}$, and the safety distance of building blocks $\varnothing_{o}^{\text {min }}$ and supply yard $\varnothing_{S u}^{\text {min }}$ are considered equal to 0 and 2 meters respectively. Also, the rate $\mu_{S u}$ is equal to 2 in all cases.

\subsubsection{Results and discussion for Case 1}

A four-story steel frame with AISC W-sections is given consisting of 273 members. The plan view of this crane layout case is illustrated in Fig. 4. Groping of the members and their weights are shown in Tables 4 and 5, respectively.

Table 6 is an abridged form of the numerical findings for the algorithms. For each algorithm, the findings encompass the best cost, average, standard deviation, and best. The results of all the algorithms are shown in this table for comparison. In addition, Table 6 is also an abridged form of the best possible solutions from 30 independent runs which point out - as for solution quality - the superior performance of the USCA approach compare with SCA and other approaches.

The summary of the best-found solutions in Table 6 indicates that the performance of the USCA method is superior to SCA and other methods in terms of solution quality.

Having and presenting Fig. 5 - which illustrates the mean convergence curve of every algorithm in the course of its iteration - assists to have a meticulous analysis

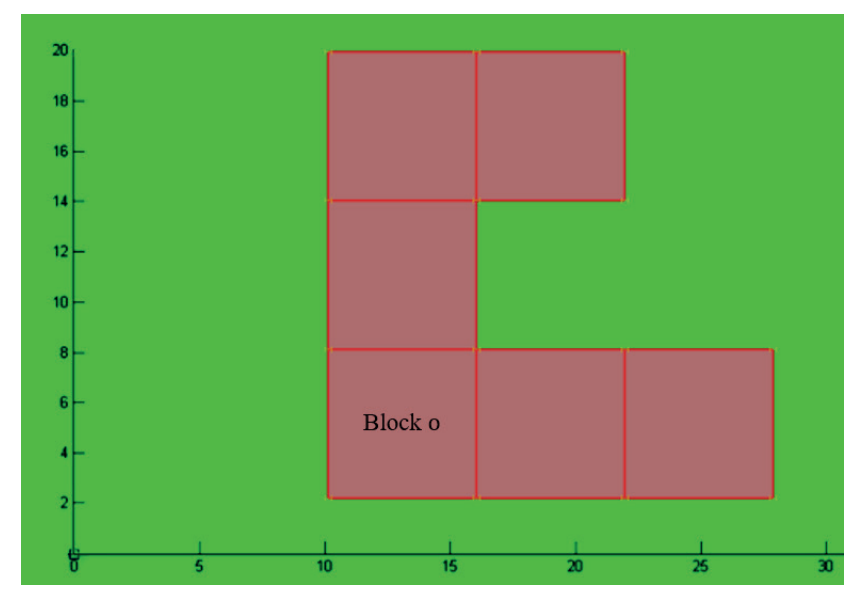

Fig. 4 Plan view of the site for Case 1
Table 4 Grouping of members in Case 1

\begin{tabular}{lcccc}
\hline Story & 1 & 2 & 3 & 4 \\
\hline Corner column & 1 & 2 & 3 & 4 \\
Side column & 5 & 6 & 7 & 8 \\
Side beam & 9 & 10 & 11 & 12 \\
Inner beam & 13 & 14 & 15 & 16 \\
\hline
\end{tabular}

Table 5 Weight of members in each group in the Case 1

\begin{tabular}{lc}
\hline Element Group & Weight of members $(\mathrm{kg})$ \\
\hline 1 & 192.5 \\
2 & 161 \\
3 & 234.5 \\
4 & 168 \\
5 & 192.5 \\
6 & 199.5 \\
7 & 175 \\
8 & 168 \\
9 & 156 \\
10 & 156 \\
11 & 126 \\
12 & 132 \\
13 & 156 \\
14 & 186 \\
15 & 132 \\
16 & 132 \\
\hline
\end{tabular}

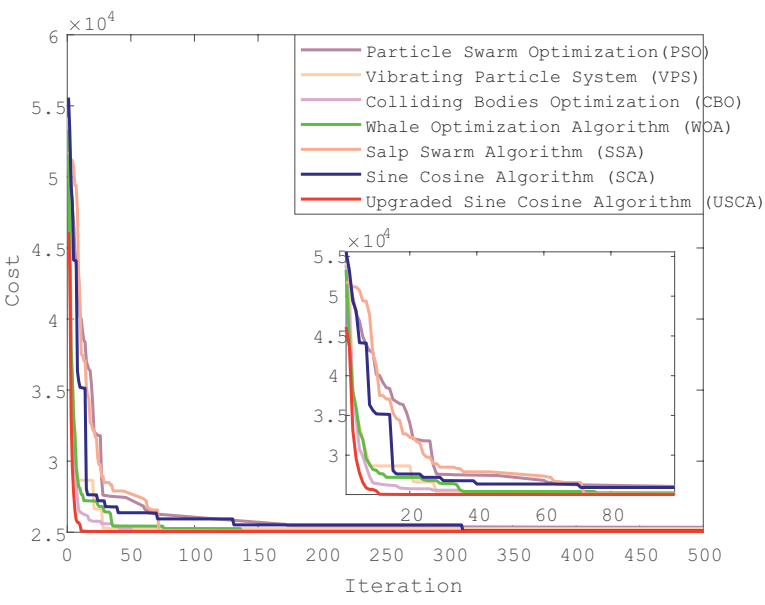

Fig. 5 Mean cost convergence curves of Case 1

and discussion about the numerical results. According to Table 6 and Fig. 6 and, it is apparent that tower crane Type 1 is selected and located in point (47.20) to supply materials from supply yard with dimensions of (5.8) where locates at centroid (15.30). 
Table 6 Comparison of the results using different algorithms for Case 1

\begin{tabular}{|c|c|c|c|c|c|c|c|}
\hline & PSO & VPS & $\mathrm{CBO}$ & WOA & SSA & SCA & USCA \\
\hline Best & 25049.4273 & 25053.0185 & 25047.5003 & 25048.0564 & 25047.5003 & 25048.6617 & 25047.5003 \\
\hline avg & 25346.6573 & 25250.5447 & 25075.5068 & 25068.7027 & 25067.4771 & 25087.0653 & 25048.1945 \\
\hline Std & 1045.845 & 922.623 & 48.3265 & 22.2254 & 45.3213 & 45.3552 & 1.36043 \\
\hline tower crane type & 1 & 1 & 1 & 1 & 1 & 1 & 1 \\
\hline$X_{C r}^{\prime}$ & 1 & 10 & 37 & 14 & 37 & 37 & 37 \\
\hline$Y_{C r}^{\prime}$ & 2 & -7 & 2 & 29 & 2 & 2 & 2 \\
\hline$X_{S u}^{\prime}$ & 28 & 27 & 5 & 19 & 5 & 5 & 5 \\
\hline$Y_{S u}^{\prime}$ & 13 & 18 & 12 & -3 & 12 & 14 & 12 \\
\hline$L_{S u}^{x}$ & 8 & 5 & 5 & 8 & 5 & 5 & 5 \\
\hline$L_{S u}^{y}$ & 5 & 8 & 8 & 5 & 8 & 8 & 8 \\
\hline
\end{tabular}

The best experimental results are shown in bold.

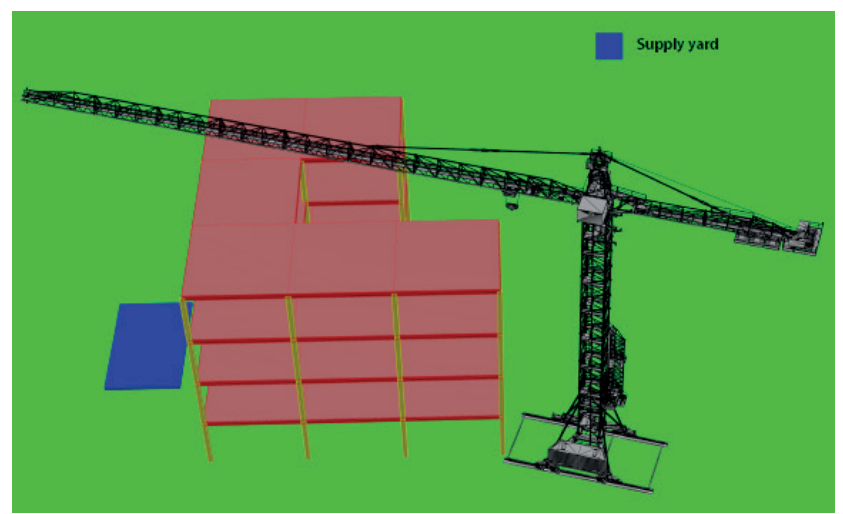

Fig. 6 Best layout of USCA for Case 1

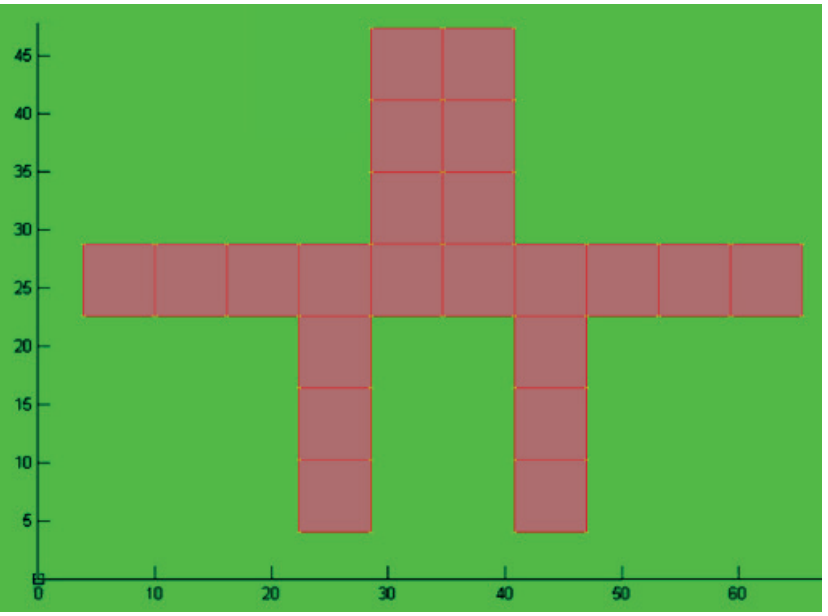

Fig. 7 Plan view of the site for Case 1

\subsubsection{Results and discussion for Case 2}

The second case study is also a four-story steel frame with AISC W-sections, which has 428 members. The plan of the crane layout case from the top view is shown in Fig. 7. Groping of the members and their weights are shown in Table 7 and Table 8, respectively.
Table 7 Grouping of members in Case 1

\begin{tabular}{lcccc}
\hline Story & 1 & 2 & 3 & 4 \\
\hline Corner column & 1 & 2 & 3 & 4 \\
Side column & 5 & 6 & 7 & 8 \\
Inner column & 9 & 10 & 11 & 12 \\
Side beam & 13 & 14 & 15 & 16 \\
Inner beam & 17 & 18 & 19 & 20 \\
\hline
\end{tabular}

Table 8 Weight of the members in each group for the Case 1

\begin{tabular}{|c|c|}
\hline Element Group & Weight of members $(\mathrm{kg})$ \\
\hline 1 & 175 \\
\hline 2 & 238 \\
\hline 3 & 185.5 \\
\hline 4 & 168 \\
\hline 5 & 238 \\
\hline 6 & 238 \\
\hline 7 & 175 \\
\hline 8 & 157.5 \\
\hline 9 & 294 \\
\hline 10 & 227.5 \\
\hline 11 & 301 \\
\hline 12 & 738.5 \\
\hline 13 & 156 \\
\hline 14 & 156 \\
\hline 15 & 156 \\
\hline 16 & 156 \\
\hline 17 & 156 \\
\hline 18 & 156 \\
\hline 19 & 132 \\
\hline 20 & 126 \\
\hline
\end{tabular}


In this part, the performance of the USCA and SCA are compared with two newly developed metaheuristic algorithms (WOA and SSA) and some distinguished metaheuristic algorithms from the literature with regard to their efficacy in analyzing a TCSLP. In order to explore the performance of the suggested USCA algorithm, we made a comparison with some known algorithms. Thus, in Table 9, there is an abridged data about the statistical information of 30 separate runs for the metaheuristic algorithms.

In Table 9, the optimum solutions of the USCA algorithm and other algorithms are shown for comparison. For all the considered algorithms, the results include the statistical results (best cost, average, and standard deviation) and best layout (tower crane location and allocation order of supply points to demand points $1,2, \ldots$, and 9 ).

In the same manner, Table 9 also shows an abridged text of the best potential solutions which designate, as far as solution quality concerned, the surpassing performance of the USCA method in comparison to other methods.

By comparison, it can be found that USCA not only outperforms SCA but also it has better performance regarding solution quality with majority of the algorithms.

Having and presenting Fig. 8 - which illustrates the mean convergence curve of every algorithm in the course of its iteration - assists to have a well-elaborated analysis and discussion about the numerical results. The optimal solution is shown in Table 9 and Fig. 9. As it can be seen from Fig. 9 and Table 9, it is apparent that tower crane Type 5 is selected and located in point (63.46) to supply materials from supply yard with dimensions of (5.8) where locates at centroid (76.74).

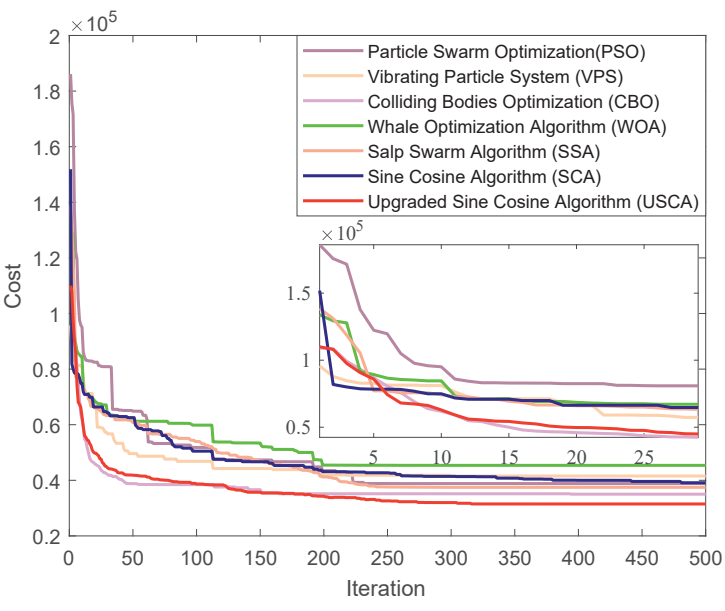

Fig. 8 Mean cost convergence curves for Case 2

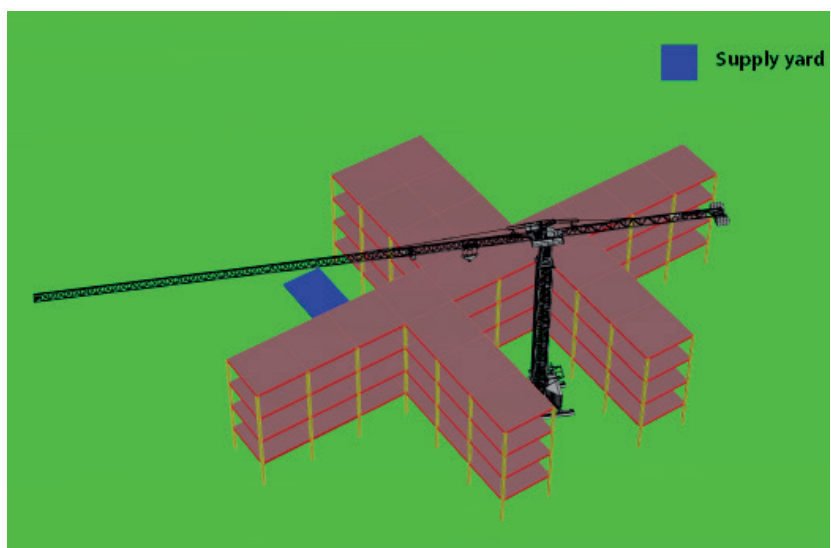

Fig. 9 Best layout of USCA for Case 2

\subsubsection{Results and discussion for Case 3}

This case study is a twelve-story steel frame with AISC $\mathrm{W}$-sections, having 376 members. The plan view of this case is presented in Fig. 10. Groping of the members and their weights are shown in Table 10 and Table 11, respectively.

Table 9 The Comparison result of algorithms for Case 2

\begin{tabular}{|c|c|c|c|c|c|c|c|}
\hline & PSO & VPS & $\mathrm{CBO}$ & WOA & SSA & SCA & USCA \\
\hline Best & 31043.0598 & 31043.0598 & 30774.1695 & 31043.0598 & 30774.1695 & 31043.0598 & 30774.1695 \\
\hline Avg & 38822.865 & 41542.1881 & 35017.2569 & 45375.1079 & 37471.3581 & 39120.0896 & 31487.2826 \\
\hline Std & 6846.233 & 8364.4197 & 5001.53 & 9676.631 & 4502.701 & 3172.423 & 2171.8856 \\
\hline Tower crane type & 4 & 4 & 5 & 4 & 5 & 4 & 5 \\
\hline$X_{C r}^{\prime}$ & 38 & 38 & 38 & 38 & 38 & 38 & 38 \\
\hline$Y_{C r}^{\prime}$ & 21 & 21 & 21 & 21 & 21 & 21 & 21 \\
\hline$X_{S u}^{\prime}$ & 57 & 57 & 51 & 57 & 51 & 57 & 51 \\
\hline$Y_{S u}^{\prime}$ & 22 & 22 & 53 & 22 & 53 & 22 & 53 \\
\hline$L_{S u}^{x}$ & 5 & 5 & 5 & 5 & 5 & 5 & 5 \\
\hline$L_{S u}^{y}$ & 8 & 8 & 8 & 8 & 8 & 8 & 8 \\
\hline
\end{tabular}

The best experimental results are shown in bold. 


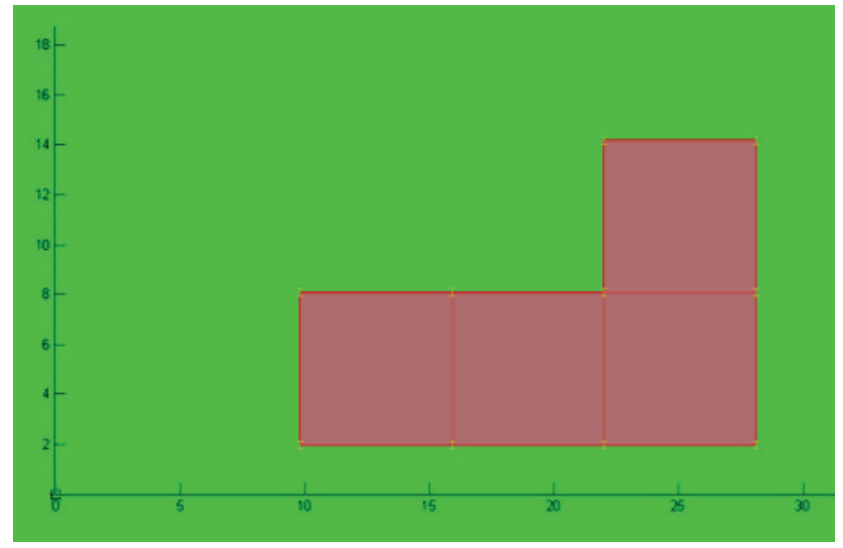

Fig. 10 Plan view of the site for Case 3

Table 10 Grouping of members in Case 1

\begin{tabular}{lcccccc}
\hline Story & $1-4$ & $3-4$ & $5-6$ & $7-8$ & $9-10$ & $11-12$ \\
\hline Corner column & 1 & 2 & 3 & 4 & 5 & 6 \\
Side column & 7 & 8 & 9 & 10 & 11 & 12 \\
Side beam & 13 & 14 & 15 & 16 & 17 & 18 \\
Inner beam & 19 & 20 & 21 & 22 & 23 & 24 \\
\hline
\end{tabular}

In this section, both benchmark algorithms and optimal solutions achieved from USCA are presented in Table 12. With regard to the best cost, the standard, and the average deviation throughout 30 simulation iterations, the optimization results are obtained to assess the precision and the stability of the benchmark algorithms, which are presented in Table 12. The results of the real case study indicate that the USCA algorithm presents more reliable solutions compared to the SCA, and it is very competitive versus other benchmark algorithms in terms of stability.

The mean convergence curves of optimization techniques are presented in Fig. 11. Investigating this figure confirms that the USCA behaves faster than other
Table 11 Weight of members in each group in the Case 1

\begin{tabular}{|c|c|}
\hline Element Group & Weight of members $(\mathrm{kg})$ \\
\hline 1 & 413 \\
\hline 2 & 353.5 \\
\hline 3 & 462 \\
\hline 4 & 346.5 \\
\hline 5 & 315 \\
\hline 6 & 595 \\
\hline 7 & 822.5 \\
\hline 8 & 696.5 \\
\hline 9 & 812 \\
\hline 10 & 773.5 \\
\hline 11 & 591.5 \\
\hline 12 & 759.5 \\
\hline 13 & 372 \\
\hline 14 & 372 \\
\hline 15 & 330 \\
\hline 16 & 360 \\
\hline 17 & 186 \\
\hline 18 & 288 \\
\hline 19 & 240 \\
\hline 20 & 270 \\
\hline 21 & 318 \\
\hline 22 & 300 \\
\hline 23 & 846 \\
\hline 24 & 210 \\
\hline
\end{tabular}

algorithms in terms of convergence speed. Fig. 12 shows the best-found solution of this paper by USCA. As can be seen from Fig. 12 and Table 12 tower crane Type 5 is assigned to a location with $X_{C r}^{\prime}$ and $Y_{C r}^{\prime}$ coordinates of 7 and 19 , respectively to supply steel frames from supply yard with dimensions (5.8) located in point (38.20).

Table 12 Comparison of the results of the algorithms for Case 3.

\begin{tabular}{|c|c|c|c|c|c|c|c|}
\hline & PSO & VPS & $\mathrm{CBO}$ & WOA & SSA & SCA & USCA \\
\hline Best & 30237.2729 & 30233.6411 & 30234.4237 & 30232.4910 & 30232.4910 & 30233.4333 & 30232.4910 \\
\hline avg & 37599.6565 & 37227.2476 & 39224.7855 & 39802.2626 & 30264.6556 & 36667.5658 & 30238.9881 \\
\hline Std & 10971.9316 & 9736.7181 & 9649.0305 & 14626.8785 & 47.4766 & 16352.0203 & 6.2586 \\
\hline Tower crane type & 5 & 5 & 5 & 5 & 5 & 5 & 5 \\
\hline$X_{C r}^{\prime}$ & 36 & 28 & 0 & 2 & 2 & 4 & 2 \\
\hline$Y_{C r}^{\prime}$ & 2 & 22 & -1 & 6 & 6 & 4 & 6 \\
\hline$X_{S u}^{\prime}$ & 12 & 18 & 28 & 33 & 33 & 33 & 33 \\
\hline$Y_{S u}^{\prime}$ & 13 & -3 & 4 & 7 & 7 & 9 & 7 \\
\hline$L_{S u}^{x}$ & 8 & 8 & 5 & 5 & 5 & 5 & 5 \\
\hline$L_{S u}^{y}$ & 5 & 5 & 8 & 8 & 8 & 8 & 8 \\
\hline
\end{tabular}

The best experimental results are shown in bold. 


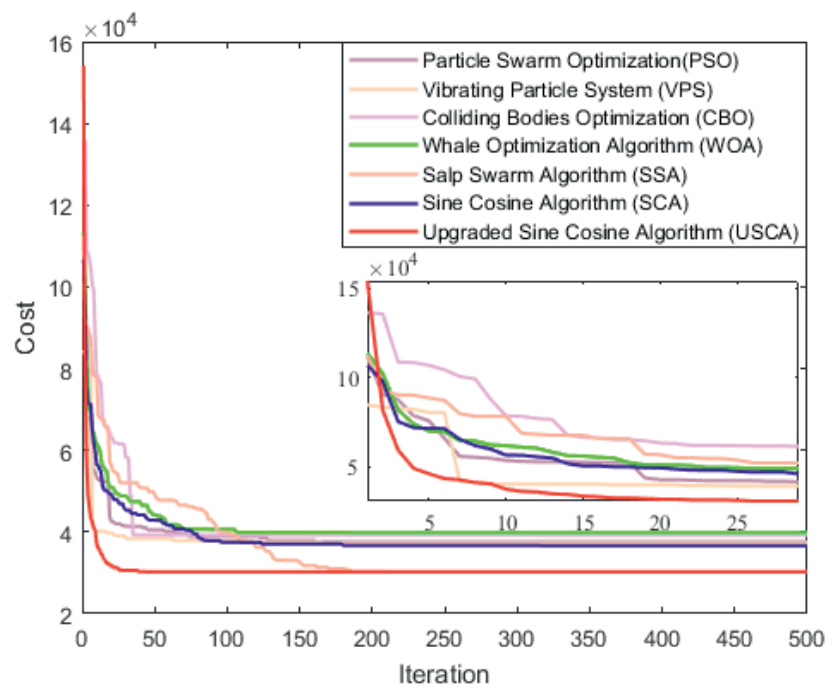

Fig. 11 Mean cost convergence curves for Case 3

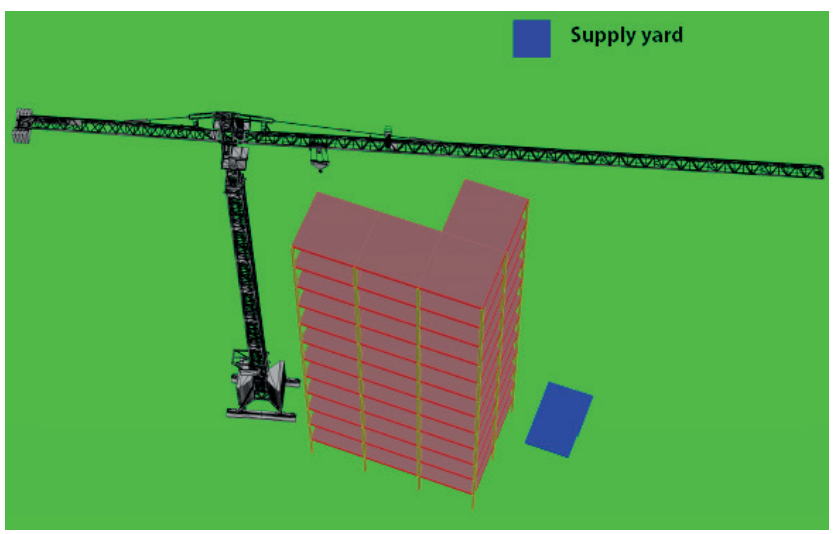

Fig. 12 Best layout of USCA for Case 3

\section{Conclusions}

From the results of the experimental studies on mathematical functions and various tower crane locating scenarios, it can be found that by adding agent memory and the HS-based side constraint approach, the performance of the SCA is improved. Also, these features have made the USCA competitive with other known or recently developed algorithms, placing USCA in the first or second rank for majority of test examples.

This paper presents a new version of sine cosine algorithm which combines a memory for best ever found results and a harmony search based local search operator. This strategy makes the algorithm avoid falling into the local optima through local search with a small probability. The experimental results showed that it is beneficial to add the local search operator to the sine cosine algorithm. Compared to other locating algorithms, USCA has more advantages like faster convergence speed and superior stability. In this paper, USCA is tested on several mathematical test functions and five TCSLP scenarios. Since only five benchmark functions have been tested, the lack of research on broader dimensions is the limitation of this research. Although, from the results of the experiment it is found that the proposed USCA algorithm produces better stability and optimization results. In this research, the USCA employed only on TCSLP, however, the application of this algorithm can future be extended for solution of other engineering problems.

\section{Compliance with ethical standards}

Conflict of interest: No potential conflict of interest was reported by the authors.

\section{Nomenclature}

\section{Abbreviations, superscripts and subscripts}

AHP Analytic Hierarchy Process

AISC American Institute of Steel Construction

ALO Ant Lion Optimizer

AM Agents Memory

AMCR Agent Memory Considering Rate

BIM Building Information Model

CBO Colliding Bodies Optimization

SELO Socio Evolution and Learning Optimization

CEOPs Construction Engineering Optimization Problems

CPA Cyclical Parthenogenesis Algorithm

$\mathrm{Cr} \quad$ Tower Crane

CSLPs Construction Site Layout Problems

CSS Charged System Search

De Demand point

ECBO Enhanced Colliding Bodies Optimization

FLDP Facility Layout Design Problem

GIS Geographic Information System

GSA Gravitational Search Algorithm

IA Ideology Algorithm

MILP Mixed Integer Linear Programming

MINLP Mixed-Integer Nonlinear Programming

PSO Particle Swarm Optimization

QAP Quadratic Assignment Problem

SCA Sine Cosine Algorithm

SSA Salp Swarm Optimization

$\mathrm{Su} \quad$ Supply point

TCSLP Tower Crane Selection and Layout Problem

TCLP Tower Crane Layout Planning

USCA Upgraded Sine Cosine Algorithm 


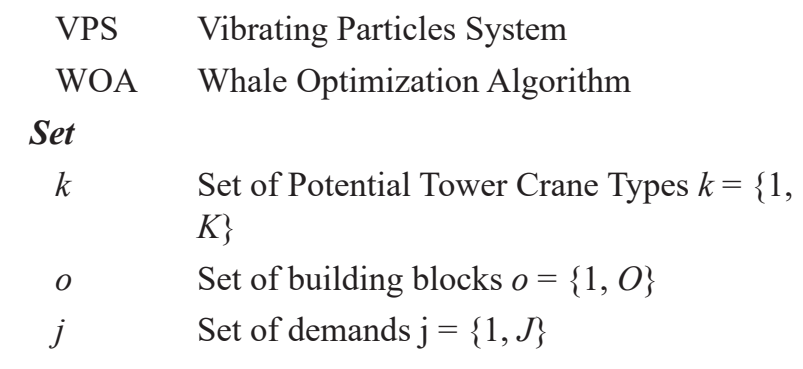

\section{Parameters}

a A constant number (here it is considered equal to 2).

$t \quad$ The current iteration in the SCA

$T \quad$ The maximum iteration number in SCA

$X_{j}, Y_{j}, Z_{j}$ Coordinates of the th demand point

$V_{k}^{v} \quad$ Hoisting velocity of the th tower crane's hook ( $\mathrm{m} / \mathrm{min})$

$V_{k}^{w} \quad$ Slewing velocity of the th tower crane's jib $(\mathrm{rad} / \mathrm{min})$

$V_{k}^{r} \quad$ Radial velocity of the th tower crane's jib (m/min)

TC Total cost

$O C_{k} \quad$ Operating cost of tower crane $k$

$F C_{k} \quad$ Fixed cost of tower crane $k$

$\mu_{S u} \quad$ Maximum permissible ratio between two aspects (sides) of the supply point $(\mathrm{Su})$ $\left(\mu_{S u} \geq 1\right)$

$A_{S u} \quad$ Area of supply yard

$\varnothing_{C r, k}^{\min }, \varnothing_{C r, k}^{\max }$ Minimum and maximum allowable distance of tower crane type $k$ from a building block (Obstacle) to attache the tower crane into the building in high rises

$X_{o}, Y_{o} \quad$ Centroids of building blocks (obstacle)

$L_{o}^{x}, L_{o}^{y} \quad$ Dimensions of building blocks (obstacles)
$\varnothing_{o}^{\min } \quad$ Minimum safety distance of obstacles or building blocks from other departments in the site
$\varnothing_{S u}^{\min } \quad$ Minimum safety distance between supply yard and other facilities in the site
$M \quad$ An arbitrary large integer number
$L^{x}, L^{y} \quad$ Dimensions of construction site

\section{Variables}

$P_{i} \quad$ The destination point's position in $i$ th dimension in the SCA process

$K_{n}^{t} \quad$ the $n$th dimension of current solution at $t$ th iteration in the SCA

$\delta_{j k} \quad$ A set of binary type variables to define material flow, which is equal to "1",

if tower Crane type $k$ transports the material supply yard, towards $j$ th demand point, and "0" if not.

$L_{C r, k}^{x}, L_{C r, k}^{y}$ Dimensions of tower crane base

$\mathrm{Ra}_{k} \quad$ Maximum jib length of tower crane type

$\mathrm{HUH}_{k} \quad$ Height of tower crane unuder hook

Weight $_{j} \quad$ Weight of material demand at point

$\mathrm{MLM}_{k} \quad$ Maximum load moment of tower crane

$\mathrm{Cap}_{k} \quad$ Maximum capacity of tower crane

$\aleph_{k} \quad$ Shows the selection of tower crane type

$X_{C r}^{\prime}, Y_{C r}^{\prime} \quad$ Centroids of the th tower crane location

$A_{S u} \quad$ The area of supply yard

$X_{S u}^{\prime}, Y_{S u}^{\prime}, Z^{S u}$ Centroids of the th supply yard

$L_{S u}^{x}, L_{S u}^{v} \quad$ Dimensions of supply yard

$T_{j k} \quad$ The actual transportation time of demand at point from supply yard by the th tower Crane type

\section{References}

[1] Sharafi, P., Mortazavi, M., Samali, B., Ronagh, H. "Interlocking system for enhancing the integrity of multi-storey modular buildings", Automation in Construction, 85, pp. 263-272, 2018. https://doi.org/10.1016/j.autcon.2017.10.023

[2] Lien, L.-C., Cheng, M.-Y. "Particle bee algorithm for tower crane layout with material quantity supply and demand optimization", Automation in Construction, 45, pp. 25-32, 2014. https://doi.org/10.1016/j.autcon.2014.05.002

[3] Crawford, B., Soto, R., Astorga, G., García, J., Castro, C., Paredes, F. "Putting Continuous Metaheuristics to Work in Binary Search Spaces", Complexity, Article ID 8404231, 2017. https://doi.org/10.1155/2017/8404231

[4] Mirjalili, S. "SCA: A Sine Cosine Algorithm for solving optimization problems", Knowledge-Based Systems, 96, pp. 120-133, 2016. https://doi.org/10.1016/j.knosys.2015.12.022

[5] Kaveh, A. "Advances in Metaheuristic Algorithms for Optimal Design of Structures", 2nd ed., Springer, Cham, Switzerland, 2017. https://doi.org/10.1007/978-3-319-05549-7

[6] Eberhart, R., Kennedy, J. "A new optimizer using particle swarm theory", In Proceedings of the Sixth International Symposium on Micro Machine and Human Science, Nagoya, Japan, 1995, pp. 39-43. https://doi.org/10.1109/MHS.1995.494215

[7] Golberg, D. E. "Genetic Algorithms in Search, Optimization, and Machine Learning. Reading", Addison-Wesley Publishing Longman Co., Boston, MA, USA, 1989.

[8] Mirjalili, S. "The Ant Lion Optimizer", Advances in Engineering Software, 83, pp. 80-98, 2015. https://doi.org/10.1016/j.advengsoft.2015.01.010

[9] Kaveh, A., Zolghadr, A. "Cyclical Parthenogenesis Algorithm: A new meta-heuristic algorithm", Asian Journal of Civil Engineering, 18(5), pp. 673-701, 2017. 
[10] Kumar, M., Kulkarni, A. J., Satapathy, S. C. "Socio evolution \& learning optimization algorithm: A socio-inspired optimization methodology", Future Generation Computer Systems, 81, pp. 252$272,2018$.

https://doi.org/10.1016/j.future.2017.10.052

[11] Huan, T. T., Kulkarni, A. J., Kanesan, J., Huang, C. J., Abraham, A. "Ideology algorithm: a socio-inspired optimization methodology", Neural Computing and Applications, (28), pp. 845-876, 2017. https://oi.org/10.1007/s00521-016-2379-4

[12] Rashedi, E., Nezamabadi-pour, H., Saryazdi, S. "GSA: A Gravitational Search Algorithm", Information Sciences, 179(13), pp. 2232-2248, 2009. https://doi.org/10.1016/j.ins.2009.03.004

[13] Kaveh, A., Talatahari, S. "A novel heuristic optimization method: charged system search", Acta Mechanica, 213, pp. 267-289, 2010. https://doi.org/10.1007/s00707-009-0270-4

[14] Kaveh, A., Ilchi Ghazaan, M. "Optimum Design of Skeletal Structures using PSO-Based Algorithms", Periodica Polytechnica Civil Engineering, 61(2), pp. 184-195, 2017. https://doi.org/10.3311/PPci.9614

[15] Kaveh, A., Vazirinia, Y. "Optimization of Tower Crane Location and Material Quantity Between Supply and Demand Points: A Comparative Study", Periodica Polytechnica Civil Engineering, 62(3), pp. 732-745, 2018. https://doi.org/10.3311/PPci.11816

[16] Huang, C., Wong, C. K., Tam, C. M. "Optimization of tower crane and material supply locations in a high-rise building site by mixed-integer linear programming", Automation in Construction, 20(5), pp. 571-580, 2011. https://doi.org/10.1016/j.autcon.2010.11.023

[17] Zhang, P., Harris, F. C., Olomolaiye, P. O., Holt, G. D. "Location Optimization for a Group of Tower Cranes", Journal of Construction Engineering and Management, 125(2), pp. 115-122, 1999. https://doi.org/10.1061/(ASCE)0733-9364(1999)125:2(115)

[18] Tam, C. M., Tong, T. K. L. "GA-ANN model for optimizing the locations of tower crane and supply points for high-rise public housing construction", Construction Management and Economics, 21(3), pp. 257-266, 2003. https://doi.org/10.1080/0144619032000049665

[19] Tam, C. M., Tong, T. K. L., Chan, W. K. W. "Genetic Algorithm for Optimizing Supply Locations around Tower Crane", Journal of Construction Engineering and Management, 127(4), pp. 315-321, 2001.

https://doi.org/10.1061/(ASCE)0733-9364(2001)127:4(315)
[20] Kaveh, A., Vazirinia, Y. "Tower cranes and supply points locating problem using CBO, ECBO, and VPS", International Journal of Optimization in Civil Engineering, 7(3), pp. 393-411, 2017. [online] Available at: http://ijoce.iust.ac.ir/article-1-305-en.html [Accessed: 10 January 2020]

[21] Wang, J., Zhang, X., Shou, W., Wang, X., Xu, B., Kim, M. J., Wu, P. "A BIM-based approach for automated tower crane layout planning", Automation in Construction, 59, pp. 168-178, 2015. https://doi.org/10.1016/j.autcon.2015.05.006

[22] Marzouk, M., Abubakr, A. "Decision support for tower crane selection with building information models and genetic algorithms", Automation in Construction, 61, pp. 1-15, 2016. https://doi.org/10.1016/j.autcon.2015.09.008

[23] Irizarry, J., Karan, E. P. "Optimizing location of tower cranes on construction sites through GIS and BIM integration", Journal of Information Technology in Construction, 17, pp. 351-366, 2012. [online] Available at: http://www.itcon.org/2012/23 [Accessed: 10 January 2020]

[24] Kaveh, A., Ilchi Ghazaan, M. "A new meta-heuristic algorithm: vibrating particles system", Scientia Iranica Transactions A: Civil Engineering, 24(2), pp. 1-32, 2017.

https://doi.org/10.24200/sci.2017.2417

[25] Huang, C., Wong, C. K., Tam, C. M. "Optimization of material hoisting operations and storage locations in multi-storey building construction by mixed-integer programming", Automation in Construction, 19(5), pp. 656-663, 2010. https://doi.org/10.1016/j.autcon.2010.02.005

[26] Mirjalili, S., Lewis, A. "The Whale Optimization Algorithm", Advances in Engineering Software, 95, pp. 51-67, 2016. https://doi.org/10.1016/j.advengsoft.2016.01.008

[27] Mirjalili, S., Gandomi, A. H., Mirjalili, S. Z., Saremi, S., Faris, H., Mirjalili, S. M., "Salp Swarm Algorithm: A bio-inspired optimizer for engineering design problems", Advances in Engineering Software, 114, pp. 163-191, 2017. https://doi.org/10.1016/j.advengsoft.2017.07.002

[28] Kaveh, A., Vazirinia, Y. "Construction Site Layout Planning Problem Using Metaheuristic Algorithms: a Comparative Study", Iranian Journal of Science and Technology - Transactions of Civil Engineering, 43, pp. 105-115, 2019. https://doi.org/10.1007/s40996-018-0148-6

[29] Kaveh, A., Ilchi Ghazaan, M. "Meta-heuristic Algorithms for Optimal Design of Real-Size Structures", Springer, Cham, Switzerland, 2018. https://doi.org/10.1007/978-3-319-78780-0 


\section{Appendix A}

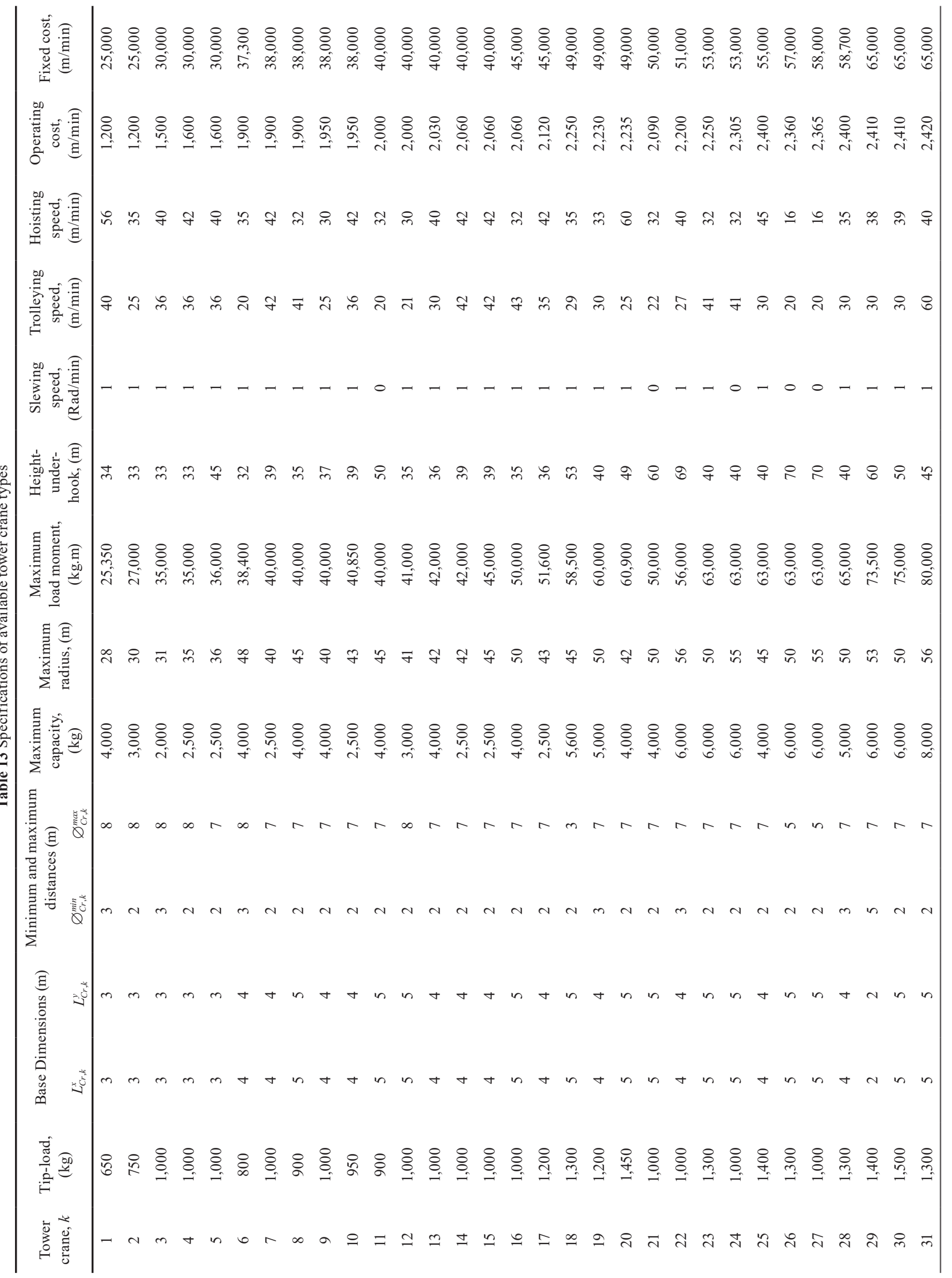




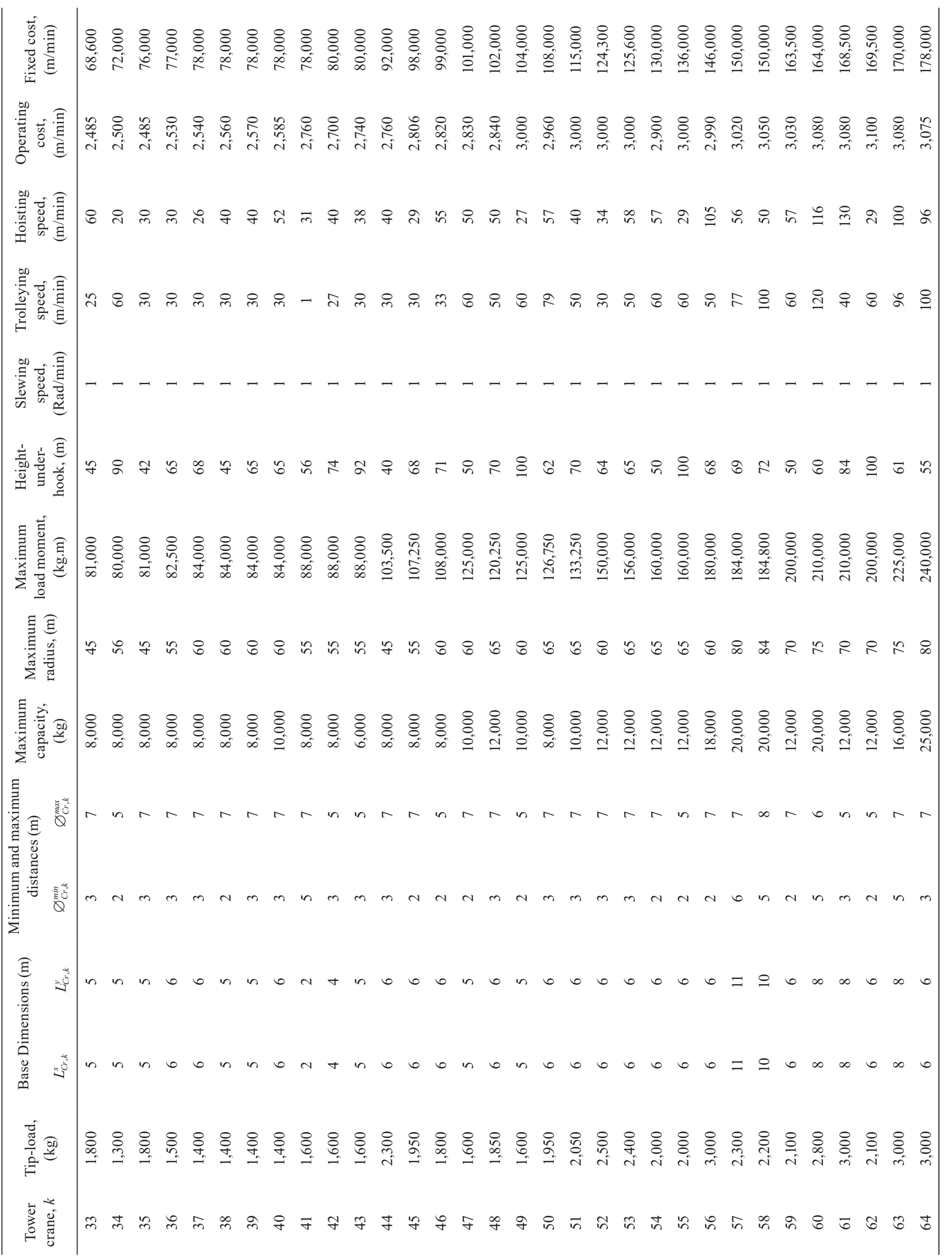




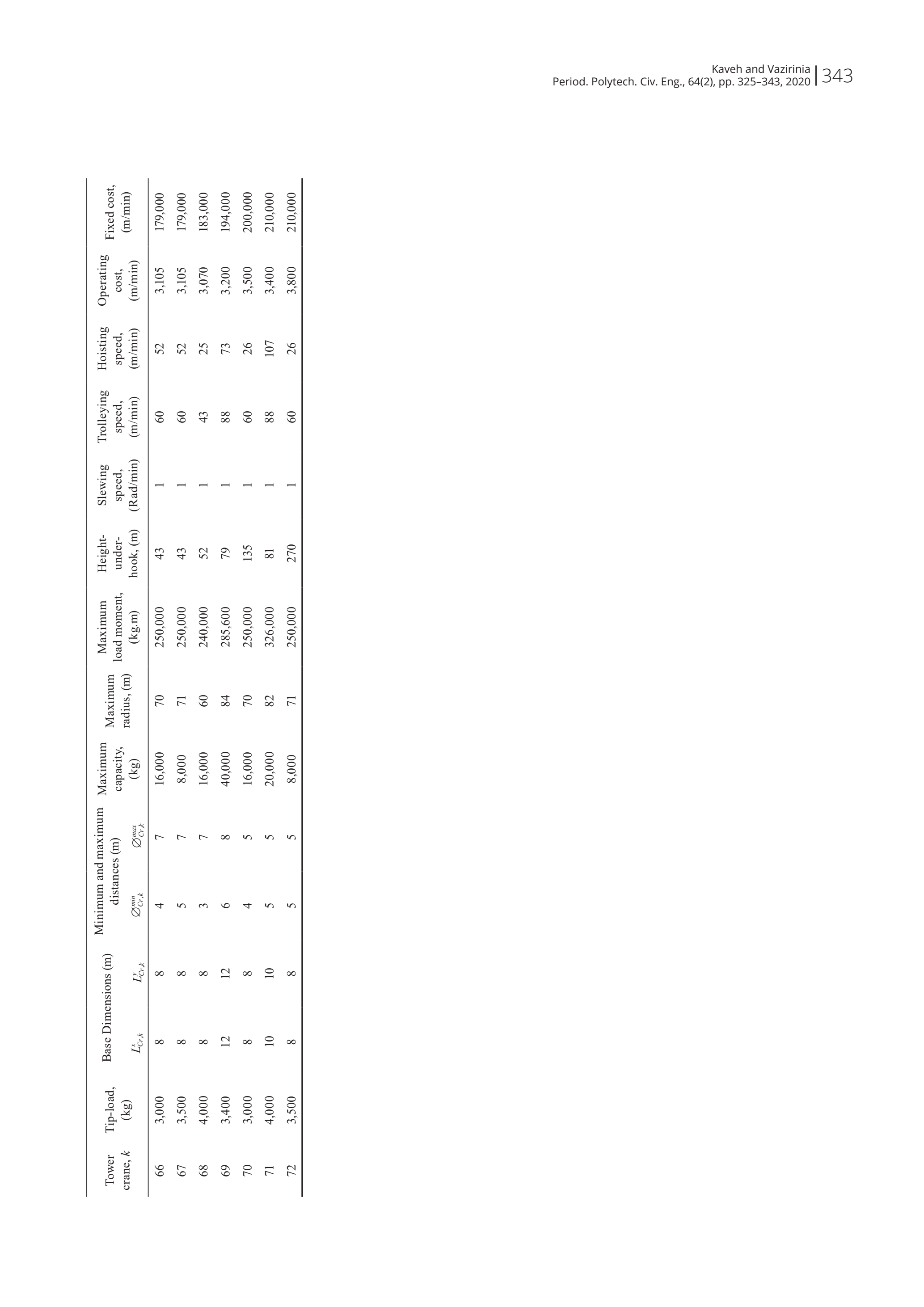

